\title{
EL PATRIMONIO INDUSTRIAL HISTÓRICO DE MÉXICO Y SUS FUENTES
}

\author{
Sergio Niccolai*
}

\section{UNA CONSTELACIÓN DE FUENTES}

$\mathrm{E}$ 1 propósito de esta contribución es atraer la atención sobre el importante y multifacético patrimonio histórico heredado de las empresas industriales (monumentos arquitectónicos y técnicos, productos, culturas del trabajo, paisajes, además de archivos documentales), resaltando que el conjunto de las fuentes que se requieren para su análisis e interpretación constituye un universo extenso y variado, que no puede limitarse a la información escrita.

El punto de vista propuesto es el de la arqueología industrial, una actividad científico-práctica relativamente reciente que se ocupa del estudio y de la conservación del patrimonio industrial histórico. Se trata de una disciplina potencialmente muy innovadora en el campo historiográfico y en el de las políticas culturales, capaz de recuperar y traducir en perspectiva moderna una de las acepciones más antiguas e interesantes del concepto de historia (historiç), es decir, investigar para comprender y hacer. ${ }^{1}$ De su carácter de "saber técnico" o "praxis racional" deriva que la arqueología industrial requiere, entre otros, también un acercamiento novedoso hacia las fuentes de información. Para que estas consideracio-

* Centro de Investigaciones Interdisciplinarias en Ciencias y Humanidades-uNAM <snicco@colmex.mx>. Esta contribución retoma e integra algunas páginas de un texto mucho más extenso presentado en el Tercer Encuentro sobre Conservación del Patrimonio Industrial Mexicano (San Luis Potosi, 11-13 de junio de 2003).

${ }^{1}$ Respecto a esta acepción, que tuvo una importante difusión en los ambientes filosóficocientíficos griegos, sobre todo en el siglo v a. C., véase Vegetti, "Scienze", 1988. 
nes resulten más transparentes, me parece oportuna una breve presentación de esta actividad. ${ }^{2}$

La arqueología industrial contempla una pluralidad de intenciones, de habilidades, de escenarios, tanto en el nivel de la investigación científica como en el nivel práctico, ya que se desenvuelve entre el estudio (de archivo, campo y laboratorio), la valoración, la conservación y, cuando esto sea factible, la reutilización de los bienes industriales históricos.

Esta verdadera multidisciplina concentra su atención en una cantidad amplia de objetos (restos materiales -edificios, máquinas, herramientas, infraestructuras, productos-, así como signos territoriales y huellas culturales); abarca todas las ramas en las cuales convencionalmente se subdivide el sector industrial (o sea, las extractivas, las de procesamiento y las de transformación) y se extiende a los medios de transporte y comunicación (ferrocarriles, barcos, puentes, telégrafos, faros, entre otros), así como al sector energético, típicos de la civilización industrial.

La actividad arqueológico-industrial mira hacia el pasado, interesándose especialmente por lo que queda de los procesos industriales mecanizados y automatizados distintivos de los últimos dos siglos, sin excluir (especialmente en las experiencias de industrialización lenta y tortuosa) unidades y formas productivas con alta intensidad de trabajo y regionalmente específicas (casos éstos representados en México por la minería de plata, los obrajes de lana, los ingenios azucareros y las manufacturas tabacaleras del periodo colonial, así como por el artesanado autóctono, la producción henequenera y la de bebidas alcohólicas, entre ellas el tequila). ${ }^{3}$ Pero, por encima de todo, esta disciplina no pierde de vista el futuro, pues busca valorizar los vestigios industriales para insertarlos como elementos vitales en las actuales dinámicas socioeconómicas y en un nuevo diseño del territorio físico y cultural.

Por sus características y perspectivas depende de muchas colaboraciones: implica la convivencia del historiador, del arqueólogo, del arquitecto, del ingeniero, del sociólogo, del antropólogo, del químico y del geólogo (así como de otras figuras profesionales científicas y técnicas), y al mismo tiempo de los empresarios, de los políticos, de los administradores locales y de los ciudadanos.

${ }^{2}$ Para una introducción a esta disciplina, consúltense Cossons, BP Book, 1993; Bergeron y Dorel-Ferré, Patrimoine, 1996; Gordon y Malone, Texture, 1997; Stratton y Trinder, Twentieth, 2000, y Tognarini y Nesti, Archeologia, 2003 (recibido después de haber redactado este texto). En relación con la experiencia industrial mexicana, Novelo, Arqueología, s. f., y Carozzi, Arqueología, 1991. Un instrumento básico es Trinder, Blackwell, 1992.

${ }^{3}$ En relación con el valor cultural y las oportunidades de reutilización de una estructura manufacturera colonial de la ciudad de México, véase el estudio de Cárdenas, Obraje, 2002. Otro ejemplo de actividad industrial específica del territorio mexicano, la henequenera, está muy bien ilustrada en Nickel, Henequén, 1995. 
Como es evidente, una actividad con este perfil requiere de una constelación de fuentes de información que resultan muy variadas por su origen, estructura, soporte y modalidades de comunicación, y de las cuales las empresas industriales son las principales generadoras -a veces de manera voluntaria, la mayor parte de las veces de manera involuntaria. Una porción de estas fuentes (no muy grande) ha empezado a organizarse en archivos formales (públicos y privados), una gran parte queda almacenada en desuso y otra parte -quizás la más interesante- sobrevive dispersa en el territorio, desorganizada y descuidada.

Recientemente -para disponer de un instrumento de orientación- intentê esbozar una tipología crítica y operativa respecto a este complejo universo de fuentes, que las reúne en cinco grupos básicos: materiales, escritas, icónicas, orales y sonoras. Todos ellos tienen gran potencial interpretativo en el plano individual, $y$ un alto valor comprobatorio una vez que se amalgamen de manera adecuada. En este texto quisiera detenerme solamente en los dos grupos más importantes: el de las fuentes materiales y el de las escritas, ilustrarlos mediante unos ejemplos significativos, en relación con el caso mexicano, y derivar en una propuesta final.

\section{FUENTES MATERIALES}

En la perspectiva del estudioso del patrimonio industrial histórico, las fuentes materiales son las prioritarias por su utilidad, tanto en la fase de reconocimiento como en la de valorización del objeto de análisis y de intervención.

Entre ellas destaca la unidad productiva entendida como el conjunto de las instalaciones dedicadas a la producción de bienes industriales y de los edificios espacialmente contiguos y técnicamente complementarios. Me gusta repetir una frase que considero de gran eficacia expresiva y que puede aplicarse a cualquier tipo de instalación industrial: "la fábrica [...] es un archivo". "Un archivo material que proporciona una pluralidad considerable de informaciones básicas sobre las razones de la localización territorial, la distribución y conformación de los espacios edificados, la disposición del sistema de máquinas y de las fuentes de energía, la existencia y características de talleres de reparación, almacenes y oficinas administrativas, la organización del trabajo, los enlaces con los mercados de materias primas y productos finales. ${ }^{5}$ Tan sólo los aspectos arquitectónicos estructurales del monumento fabril -restablecidos, según los casos, a través de la excavación selectiva de corte topográfico (sincrónico) o

4 Tavone, "Introduzione", 1989, p. 1.

${ }^{5} \mathrm{Al}$ respecto es muy interesante Stratton y Trinder, Book, 1997. 
estratigráfico (diacrónico), de la reconstrucción de las planimetrías, del examen de muros, pisos y otros componentes- señalan su trayectoria vital. ${ }^{6}$ Es decir, las soluciones elegidas originalmente en la adecuación de estructuras anteriores (molinos, manufacturas, almacenes) a las industriales modernas, aquellas adoptadas en la instalación de maquinaria pesada importada y en la utilización de fuentes energéticas locales, además de los obstáculos y las respuestas frente a las mutaciones en los sistemas técnicos. En unos casos, a partir de una lectura atenta de los trazos estéticos, arquitectónicos y de ingeniería de la unidad productiva, se puede inferir la existencia de los modelos constructivos de referencia, lo que representa una pista importante para comprender los niveles de originalidad, imitación y adaptación experimentados por las estructuras industriales mexicanas. A menudo, sobre todo en el siglo XIX, la arquitectura de la fábrica (al igual que su nombre) refleja una perspectiva dualista interno/externo, útil/bello, funcional/formal en una yuxtaposición de mensajes hacia los trabajadores, los mercados, el mundo político y la sociedad en general. En particular, su estructura externa (geometrías relativamente sencillas con fachada alta y solemne, elementos decorativos circunscritos, muros perimetrales imponentes, dispositivos de defensa) define la sólida presencia territorial de la empresa y proyecta un claro mensaje político-social basado en la disciplina y la moralidad pública, la formación del buen ciudadano y la construcción de la conciencia nacional. Al respecto, es interesante recordar que tanto en el imaginario de testigos contemporáneos como en la actualidad, estas fábricas son consideradas a veces como palacios, a veces como catedrales, a veces como castillos industriales. ${ }^{7}$

En esta misma perspectiva, un documento material que amerita una lectura particularmente atenta y que sugiere pistas significativas para interpretar el fenómeno industrial mexicano es el microcosmo fábrica-aldea-casa patronal (establecimiento industrial, más viviendas, escuela, iglesia, jardín, teatro, tiendas, hospital, cementerio) típico del siglo XIX, en particular en el caso de la localización rural o periférica de la unidad productiva. $\mathrm{El}$ análisis detallado de este mundo socioeconómico en escala reducida debe dirigirse tanto a la coincidencia de espacio productivo y espacio residencial, como a la jerarquía (y características) de los diferentes espacios residenciales: las casas de los propietarios, de los administradores, de los técnicos, del personal de confianza y de los obreros. Entre los segmentos más interesantes de este

${ }^{6}$ Para una amplia introducción a la temática de las arquitecturas industriales y a su reutilización véase Aguilar, Arquitectura, 1998. Para un documento histórico relativo a los criterios de edificación industrial, Case, Factory, 1919.

${ }^{7}$ Para una visión general, Sande, Industrial, 1978. Para un ejemplo mexicano, relativo a los ingenios azucareros, Mentz, Haciendas, 1997. 
microcosmo industrial están el caserío obrero - del cual hay que investigar dimensiones, sistemas de ventilación, dotación de agua potable, servicios higiénicos y mobiliario-, la escuela, el hospital, la iglesia (con sus ex votos) y el cementerio. Por su parte, las murallas, las púas, las torretas señalan tanto una afirmación del derecho de propiedad como una actitud temerosa hacia adentro y hacia afuera. A menudo la fábrica aparece como una fortaleza donde se resguardan los secretos productivos, los insumos y las mercancías, y el dueño se asegura de que los operarios no olviden que su libre decisión de estar allí tiene ciertos límites. Es probable que en unos casos aquellos resguardos sean manifestaciones de las actitudes paternalistas de los propietarios que suponen experimentar un proyecto filantrópico y progresista a favor de las clases subalternas. En efecto, en el caso mexicano es todavía una tarea pendiente desenmarañar las relaciones entre ideales utopistas y contextos coercitivos, creación del ambiente moralmente sano para la mejora de la familia obrera y control de tipo señorial. Falta conocer mejor las finalidades de la colonia obrera, los modelos en los cuales se inspiraban, los mecanismos de adaptación, las sociabilidades cotidianas dominantes (entre las cuales deben subrayarse las étnicas y las de género). $\mathrm{El}$ análisis de los espacios arquitectónicos, de sus funcionalidades y modificaciones en el tiempo, podrá ayudar a discriminar entre el discurso ideológico (consignado en muchas fuentes escritas y a menudo plasmado en las fachadas) y la realidad inclemente del trabajo industrial. Al mismo tiempo este análisis permitirá establecer la vigencia del modelo microcósmico frente al distanciamiento entre espacio productivo y espacio habitacional experimentado, sobre todo, desde la segunda mitad del siglo XX.

Otra fuente material privilegiada es el territorio (como ámbito espacial condicionado por un núcleo industrial). Con la intensificación del proceso de industrialización, la fábrica (junto con los centros extractivos y las redes de transporte) se transformó en elemento característico de muchos paisajes regionales, lo que a menudo dio origen a una tensión constante entre el mundo productivo y el entorno (humano y natural). Para entender esta redefinición del paisaje y esta tensión es muy útil una observación directa (de superficie, altura, aérea) del ambiente industrial, siguiendo rastros de cambios y persistencias. ${ }^{8}$ En primer lugar, mediante el análisis de los signos espacialmente no coincidentes - la mayor parte de las veces en superficie, a veces semienterrados o completamente enterrados- conservados por el territorio (edificaciones, fragmentos de maquinaria y dispositivos, hasta capas de los desperdicios de la producción) es posible detectar una serie de continuidades estructurales o funcionales entre diferentes tipos de unidades eco-

${ }^{8}$ Para un acercamiento a la temática del contexto territorial, véase Castillo, Entorno, 1997. Para un estudio de caso, Alfrey y Clark, Landscape, 1993. 
nómicas y etapas productivas, en particular entre sistemas técnicos, energéticos y de transporte. En segundo lugar, las huellas territoriales (entre las cuales destaca la vegetación) son buenas guías para proceder a una mejor interpretación de la localización industrial y de los cambios en el uso del suelo. Por un lado, aunque en México la situación semirrural o semiurbana parece haber predominado durante mucho tiempo, el examen territorial podrá confirmar (o no) en qué grado y aspectos la "fábrica en el campo" o la "fábrica en la ciudad" representan soluciones alternativas respecto a los aspectos arquitectónicos, del sistema técnico y energético, de las relaciones sociales internas y de los intercambios externos. Por otro lado, se podrá considerar en qué medida fenómenos como el abandono de asentamientos industriales responden a razones naturales (sequías e inundaciones) o humanas (expansión de actividades productivas alternativas, deforestación y mala administración de los recursos). Finalmente, quizá uno de los aspectos más relevantes, el territorio detiene (a veces en forma de heridas abiertas, a veces en forma de toques de jardinero) pruebas tajantes de las modalidades, mecanismos e intensidad a través de los cuales los principales fenómenos vinculados con la industrialización -multiplicación y expansión de los centros urbanos (con incrustación de unidades productivas originariamente rurales o periféricas), crecimiento demográfico, expansión de la superficie agrícola, aumento exponencial en el consumo de bienes finales y recursos naturales, contaminación e invasión de basura no biodegradable- transformaron un espacio, hasta entonces relativamente estático, y provocaron en algunos casos rupturas ecológicas irreversibles. ${ }^{9}$

La lista de las fuentes materiales sería muy larga e incluiría la maquinaria y las herramientas, las materias primas, los productos, los residuos de la producción, los medios de enlace y acceso a sistemas energéticos, de transporte y comunicación. En resumen, y como resulta evidente, aquí se hace referencia a aquellos archivos materiales muertos que representan el legado de la empresa industrial una vez que ésta cierra sus actividades, fracasa, se reestructura o cambia su ubicación espacial. Entonces, de lo que era un agregado de estructuras, infraestructuras, recursos naturales y humanos, bienes de diferente característica física y valor comercial (un patrimonio activo) solamente perdura, a veces durante décadas o siglos, un conjunto residual, obsoleto desde el punto de vista económico y social (un patrimonio histórico inactivo). En un gran número de casos eso es lo único que queda para poderse acercar a la fisonomía y a la trayectoria de

${ }^{9}$ Nada más obsérvese cómo cambia el paisaje con la presencia de instalaciones productivas de grandes dimensiones (una fundición, una refineria o un parque industrial), donde hay actividades extractivas a cielo abierto, con el tendido de pilares y periféricos para el transporte de material del área minera a las haciendas de beneficio o con el tejido de cables eléctricos cerca de un área industrial moderna. 
las empresas industriales. Allí es donde puede encontrarse una serie de testigos taciturnos pero confiables.

Para finalizar estas consideraciones sobre las fuentes materiales me parece oportuno subrayar algunos aspectos generales que sustentan la reflexión de conclusión, es decir, los riesgos que amenazan a estos acervos, fundamentales para entender el pasado industrial y para valorizarlo: a) la acción del tiempo sobre los materiales y las estructuras; b) la dinámica socioeconómica en cuanto a que a menudo estas fuentes representan un obstáculo frente a los proyectos, privados o públicos, que implican una reorganización del territorio; c) el alto impacto ambiental de los restos de las edificaciones y de los desperdicios productivos acumulados.

\section{FUENTES ESCRITAS}

Después de las materiales, el segundo grupo de fuentes privilegiado por el estudioso del patrimonio industrial es el de las escritas. Esto se debe a su cantidad y riqueza informativa (explicable por el hecho de que la civilización industrial privilegió la comunicación escrita), a su resguardo organizado en archivos públicos y privados y a la experiencia adquirida en su manejo. ${ }^{10}$

En primer lugar, es necesario referirse a la documentación producida por las empresas y los empresarios, cuyo rescate y conservación es fundamental para comprender una porción sustancial del pasado industrial, tanto en sus vertientes propiamente económicas como en las culturales. Aunque disponible en escasas proporciones para las grandes empresas y casi ausente para las pequeñas, esta documentación (en particular los libros de contabilidad, los presupuestos, los inventarios, la correspondencia, las actas de las asambleas) permite avanzar en complicados problemas interpretativos, esenciales también para quien se ocupa de la valoración de la herencia industrial mexicana. Para dar un ejemplo significativo, es suficiente referirse a los inicios de la estructura industrial mecanizada, con las tantas cuestiones abiertas relativas a las continuidades con las manufacturas coloniales, a las motivaciones para las primeras inversiones en fábricas modernas, a los mecanismos de financiamiento, al carácter económicamente racional de estas elecciones en un contexto económico, político, social e institucional extremadamente complejo. En los pocos casos en los cuales es posible estudiar los papeles de una empresa, el análisis del comportamiento particular proporciona pistas para enten-

${ }^{10}$ Para una introducción a la variedad de las fuentes escritas, véase Samaran, Histoire, 1961; Cipolla, Historia, 1991, y Fontana, "Storia", 1989. 
der lo que los pocos datos cuantitativos agregados disponibles y las inferencias estadísticas no pueden más que ignorar. Pero en esta documentación hay mucho más para los que se interesan en la herencia industrial. Los archivos de empresa conservan registros importantes sobre constitución de sociedades industriales, ciclos productivos, tipos de mercancías, compra de insumos y venta de bienes intermedios o finales, arrendamiento de terrenos y fuentes de energía; complementan los datos de las empresas de transportes respecto a la localización de líneas y estaciones, tipo de vehículos y puntos de enlace, cargas y destinos de insumos y mercancías; en unos casos conducen a la identificación de los constructores de las plantas fabriles, de los criterios estilísticos y políticos para la adopción de una determinada solución arquitectónica, de las razones técnicas de edificación, modificación y reestructuración. Para finales del siglo XIX y el XX adquieren siempre más importancia las actas de los consejos de administración y de las asambleas generales de los socios, los reportes anuales, así como la correspondencia y las circulares de las oficinas técnicas y administrativas.

Entre la documentación de las empresas, los inventarios ocupan una posición central para conocer la dotación de materias primas, maquinaria y productos finales. Si los inventarios más útiles, los elaborados periódicamente por los administradores de la empresa, son bastante raros hasta finales del siglo XIX (una excepción la constituyen algunas empresas mineras y azucareras), se dispone de inventarios que se elaboran en ocasiones especiales, como una quiebra, una venta, un traspaso o post mortem del propietario. $\mathrm{Al}$ igual que en las pólizas de seguro, en estos inventarios -que se incluyen en las actas testamentarias, de compraventa o de quiebra y cuyas minutas generalmente se localizan en los archivos de los notarios- pueden encontrarse las estimaciones del valor de la planta productiva, de los demás inmuebles, del equipo técnico y de las existencias. Cuando se dispone de inventarios realizados con criterios homogéneos para momentos distintos es posible proceder a la estimación seriada de la composición del capital y de la dotación de maquinaria, lo que facilita apreciar el desarrollo estructural y técnico, y sobre esta base la estabilidad de la unidad productiva.

Otro grupo de fuentes, la literatura de empresa (estatutos, reglamentos, boletines periódicos, publicaciones conmemorativas, avisos, carteles, folletos instructivos, prontuarios) otorga mucha información sobre fundadores, cambios societarios, composición de los consejos de administración, así como sobre maquinaria, racionalización de los procesos productivos, objetivos económicos generales, ganancias e inversiones, datos que en conjunto favorecen una valoración histórica de la empresa industrial. A menudo esta folletería -junto con los libros de raya y las fichas de los empleados- se revela como una especie de manifiesto de las 
relaciones patrón-obrero y de su imagen ideal, o sea, un indicio de gran utilidad para empezar un retrato de los principales actores del universo industrial.

Por su parte, los documentos de las familias de los empresarios -entre los cuales vale señalar originales o copias de la correspondencia de las empresas, epistolarios privados, diarios personales y recortes de periódicos- otorgan datos sobre un amplio radio de cuestiones: el peso de la unidad industrial en el contexto de las actividades económicas de la familia; la posición de la estructura familiar en la gestión empresarial; los préstamos internos, los planes de expansión y las alianzas interfamiliares; la diversificación de los negocios y el origen de los capitales; juicios y prejuicios sobre los socios y los empleados; el peso asignado a la innovación técnica y a la instrucción de los sucesores; las relaciones entre fabricantes y universo sociopolítico inmediato; el desempeño en cargos políticos, administrativos, sociales, militares o eclesiásticos de parte del empresario y de sus familiares; las relaciones estables con arquitectos, ingenieros, diseñadores $y$ técnicos de confianza." $\mathrm{O}$ sea, los papeles familiares permiten reconstruir la fisonomía tanto de los pequeños empresarios como de las dinastías empresariales, y ubicar estos actores en el contexto económico, político y social. Otra perspectiva, a veces más reservada y sincera, para entender las peculiaridades del proceso de plasmación de una cultura industrial moderna en el territorio mexicano.

Datos descriptivos en cuanto a las unidades productivas particulares y sugerencias importantes respecto al papel que los privados asignaban a la producción industrial y a las innovaciones técnicas en función de la expansión de sus negocios y del progreso económico y social general se encuentran también en la documentación (a menudo fragmentada en diferentes archivos) de las asociaciones de industriales y comerciantes, desde los consulados de comerciantes coloniales hasta las juntas de industria de la primera mitad del siglo XIX y las cámaras industriales del siglo $\mathrm{XX}$.

Un valioso complemento de la documentación de las empresas y de los empresarios lo representan los documentos notariales y bancarios. Los documentos notariales (contratos, inventarios, hipotecas, donaciones, actas matrimoniales, testamentos) son de enorme importancia-gracias a

"Un caso muy interesante es el de la destacada dinastía empresarial Rul y Azcárate, activa en negocios mineros, agrícolas, ganaderos y también industriales del área centro-norte, y de la cual el Archivo General de la Nación conserva una abundante documentación que abarca desde 1728 hasta 1938. Para el periodo 1890-1940, un caso sobresaliente es el del empresario Juan F. Brittingham (director de la empresa jabonera La Esperanza, fundador de Cementos Hidalgo y Vidriera Monterrey), cuyos papeles personales y familiares actualmente están custodiados en el Archivo Histórico Papeles de Familia de la Universidad Iberoamericana-Laguna (Torreón, Coahuila). 
su carácter descriptivo, cuantificado, estandarizado y, en cierta forma, seriado- para estudiar la constitución de las sociedades industriales, la ubicación de sus plantas productivas, la distribución del capital inicial y de sus incrementos periódicos; la compraventa o arrendamiento de terrenos, inmuebles, máquinas, insumos y fuentes energéticas; los mecanismos crediticios; los tipos y el valor de los bienes muebles e inmuebles en caso de quiebra, litigios entre socios y disoluciones; las alianzas de negocio y las estrategias matrimoniales; el encargo de obras arquitectónicas, técnicas, decorativas o artísticas.

A su vez, la documentación de los bancos (y de otros organismos crediticios) no se limita a confirmar los datos o llenar las lagunas de los archivos privados o públicos respecto a los flujos del capital de inversión. Sobre todo desde las últimas dos décadas del siglo XIX los bancos (grandes y pequeños, privados y públicos, locales, nacionales e internacionales) participaron directamente o mediante diferentes tipos de servicios de financiamiento en el surgimiento y consolidación de muchas empresas industriales, así como en el apoyo a los planes públicos de creación de estructuras e infraestructuras económicas. Por su naturaleza, esta presencia generó una serie de testimonios escritos, normalmente muy precisos y claros, que ofrecen informaciones detalladas sobre las plantas productivas y su potencial técnico, las perspectivas de las empresas particulares y una valoración contemporánea del fenómeno industrial en el contexto socioeconómico ampliado.

También en el caso de las fuentes escritas, las mencionadas anteriormente constituyen únicamente algunos ejemplos. La lista debería abarcar tanto la documentación escrita producida afuera de las empresas industriales, como la que proviene de la administración económica pública, las empresas de servicios, las asociaciones sindicales y profesionales, las instituciones médicas y de seguro, los registros parroquiales y civiles, los catálogos de mercancías, los manuales técnicos, las patentes de invención, entre otros.

\section{HaCIA UN NUEVO ESCENARIO DE COlaboRACIONES}

¿Qué se puede concluir a partir de estas rápidas reflexiones sobre las fuentes materiales y las escritas, pero especialmente sobre las primeras? En primer lugar, me parece evidente que la riqueza informativa, las potencialidades interpretativas y las características de este variado conjunto de fuentes constituyen una base sólida para esbozar una invitación a una extensión de los horizontes de todos aquellos que se interesan, por una $\mathbf{u}$ otra razón, en la conservación de la memoria de las empresas y del patrimonio cultural de un determinado territorio. 
La importancia de preservar y organizar adecuadamente las fuentes escritas, y los archivos que las contienen, es indiscutible y representa una tarea de gran utilidad, no sólo para los estudiosos de la historia económica y de la herencia industrial, sino, a menudo, también para que las empresas actualmente activas puedan definir la proyección de su imagen hacia la sociedad y los mercados, así como orientar sus planes de crecimiento y reestructuración. ${ }^{12}$

En el caso de las fuentes materiales (edificios, maquinarias, dispositivos técnicos, productos, contextos ambientales) cabe una pregunta: ¿por qué los historiadores, los economistas, los sociólogos, los curadores de archivos y museos, los empresarios y los políticos, además de los ciudadanos que pagan los impuestos, deberían considerar importante contribuir al rescate (parcial o integral) de los vestigios físicos del universo industrial histórico? Vestigios que en muchos casos están en condiciones lastimosas, estorban los proyectos de desarrollo económico y urbanístico, se alejan de los criterios estéticos convencionales, producen efectos contaminantes e incluso traen malos recuerdos. La respuesta también en este caso resulta bastante simple.

La conservación de los restos del universo industrial histórico tiene doble valor cultural: por un lado, permite rescatar un fragmento significativo y muy descuidado del pasado mexicano $\mathrm{y}$, por lo tanto, apreciar mejor sus peculiaridades; por otro, constituye una forma de protección de uno de los principales grupos de fuentes útiles para el estudio y la interpretación del pasado socioeconómico local. Con frecuencia, estas fuentes son las únicas que sobreviven o que están al alcance de los estudiosos. Por ello, son los únicos testigos que pueden ofrecer una información, aunque fragmentaria y opaca, para redibujar mentalmente y entender momentos y situaciones puntuales de la trayectoria industrial de este país, en muchos aspectos muy originales y distintivos en el panorama mundial. Pero también en el caso de que se disponga de abundantes fuentes escritas, la fuente material se revela como una compañera atrevida e inteligente, dotada de una función complementaria y comprobatoria sorprendente.

$\mathrm{Al}$ parecer tenemos buenas razones para ocuparnos de proteger los vestigios físicos de las empresas industriales junto con su documentación escrita. Si esto es cierto, resulta necesario impulsar dos operaciones interrelacionadas: a) conservar y organizar los diferentes tipos de fuentes de información como condición para reconocer, analizar y valorizar el patrimonio industrial histórico; b) rescatar y salvaguardar racionalmente

12 Para una guia detallada respecto de la extensa red de archivos históricos y museos de empresa en Italia (que ya rebasa el centenar de unidades), véase Touring, Turismo, 2003 (edición bilingüe italiano/inglés). 
los bienes que conforman el patrimonio industrial histórico como oportunidad para ampliar el abanico de las fuentes útiles, y así preparar un acercamiento más completo al pasado de la economía y elaborar interpretaciones mejor fundamentadas.

Estas operaciones requieren de algunas condiciones básicas: a) una modificación en los métodos de la investigación y de la docencia, así como un cambio en las sensibilidades de los actores y usuarios de la conservación documental; $b$ ) una alianza operativa entre los especialistas en la organización de archivos históricos y museos, los historiadores de la economía, los expertos en el estudio de los residuos materiales y culturales de las empresas y, por supuesto, las empresas mismas.

En mi opinión, las potencialidades de estos cambios y de estas alianzas son muy interesantes. Entre los efectos que podrán generar pueden señalarse: a) un reconocimiento innovador de lo que son los bienes culturales históricos (entre los cuales tienen un lugar relevante los de las empresas, con sus diferentes tipos de archivos); $b$ ) una democratización real de las concepciones actuales de la cultura $y$, por ende, de la herencia cultural; c) colaboraciones efectivas y operativas entre las profesiones académicas, las empresas y los ciudadanos; $d$ ) una presencia más activa de esta masa crítica en el diseño de las políticas dirigidas a la valorización del patrimonio cultural y a la construcción de identidades colectivas; $e$ ) finalmente, importantes oportunidades de desarrollo socioeconómico con bajo impacto ecosistémico, en especial en el sector de los servicios turístico-culturales. ${ }^{13}$

En síntesis, esta propuesta sugiere la necesidad de desenterrar aspectos olvidados de la herencia cultural del territorio mexicano y de sus poblaciones, abrir espacios a nuevos significados del pasado y de sus testimonios, inventar formas más agradables, ágiles y eficaces de preservar la memoria colectiva. Entre las primeras consecuencias está la posibilidad de sanar miopías culturales notables, remediar hábitos algo escleróticos en las políticas de conservación, enfrentar las fracturas, hoy tan evidentes y peligrosas, entre el mundo intelectual, el mundo económico activo y la mayoría de los consumidores finales de los bienes y servicios culturales. Pero, en una perspectiva de largo alcance, la meta es vincular el rescate y la valorización de la información y de los bienes históricos con un diseño innovador de los espacios naturales y culturales, parte esencial de una nueva arquitectura de las convivencias. Es mi convicción que este tipo de proyectos hará posible enfrentar mejor el escenario de las profundas y rápidas transformaciones económicas, sociales y

${ }^{13}$ En relación con las diferentes oportunidades de desarrollo de tipo turístico-cultural, se remite a Herrero, Turismo, 2000, y Gutiérrez-Cortines, Desarrollo, 2002. 
culturales que estamos experimentando, y sobre todo de las que se están vislumbrando. Así es como comenzaríamos a recuperar creativamente todo el sentido de la historíç antigua: investigar para comprender y hacer.

\section{BibliografíA}

Aguilar Civera, Inmaculada, Arquitectura industrial: concepto, método y fuentes, Valencia, Diputación de Valencia/Museu d'Etnologia, 1998.

Alba Vega, CARLos (coord.), Historia y desarrollo industrial de México, México, Confederación de Cámaras Industriales de los Estados Unidos Mexicanos, 1988.

Alfrey, Judith y CATHERIne ClARK, The Landscape of Industry. Patterns of Change in the Ironbridge Gorge, Londres/Nueva York, Routledge, 1993.

BERgERON, LOUIS Y GRACIA DOREL-FERRE, Le patrimoine industriel, un nouveau territoire, París, Liris, 1996.

Cárdenas Gutiérrez, Salvador, El obraje de Mixcoac en el siglo xVIII, México, Universidad Panamericana, 2002.

Carozzi, Gigliola, La arqueología industrial, México, Departamento de Arquitectura y Urbanismo-Universidad Iberoamericana, 1991.

Case, Willard L., The Factory Buildings, Nueva York, Industrial Extension Institute, 1919.

Castillo Ruiz, José, El entorno de los bienes inmuebles de interés cultural. Concepto, legislación y metodologías para su delimitación. Evolución histórica y situación actual, Granada, Universidad de Granada/Instituto Andaluz del Patrimonio Histórico, 1997.

CIPOlla, CARlo M., Entre la historia y la economía. Introducción a la historia económica, Barcelona, Crítica, 1991.

Comité Mexicano para la Conservación del Patrimonio Industrial, Memoria. Segundo encuentro nacional para la conseroación del patrimonio industrial. El patrimonio industrial mexicano frente al nuevo milenio y la experiencia latinoamericana (Monterrey, 6-8 de junio de 2001), Aguascalientes, Universidad Autónoma de Aguascalientes/ Universidad de Guadalajara/Comité Mexicano para la Conservación del Patrimonio Industrial, A. C./ConAculta/Museo Nacional de Ferrocarriles Mexicanos/Gobierno del Estado de Aguascalientes, 2002.

Cossons, NeIL, The BP Book of Industrial Archaeology, Newton Abbot, David \& Charles, 3a. ed., 1993.

Daumas, Maurice (dir.), Histoire générale des techniques, París, Presses Uni versitaires de France, 1996, 5 tomos.

FontANA, GIOVANNI LUIGI, "Tra storia dell'industria e archeologia industriale: le fonti documentarie" en BERNARDETTA RICATTI y FRANCESCO TAVONE (coords.), Archeologia industriale e scuola, Florencia, Manzuoli/Casale Monferrato, Marietti, 1989, pp. 15-41. 
Gordon, Robert B. y Patrick M. Malone, The Texture of Industry. An Archaeological View of the Industrialization of North America, Nueva York, Oxford University Press, 1997.

Gutiérrez-Cortines Corral, Cristina (ed.), Desarrollo sostenible y patrimonio histórico y natural. Una nueva mirada hacia la renovación del pasado, Santander, Fundación Marcelino Botín, 2002, 2 tomos.

Herrero Prieto, LuIs CÉSAR (coord.), Turismo cultural: el patrimonio histórico como fuente de riqueza, Valladolid, Fundación del Patrimonio Histórico de Castilla y León, 2000.

Hudson, Kenneth, World Industrial Archaeology. An Introduction, Cambridge, Cambridge University Press, 1979.

León, Martha E. (coord.), Caminos de hierro, México, Secretaría de Comunicaciones y Transportes/Ferrocarriles Nacionales de México, 1996.

MeNTZ, BRigida von et al., Haciendas de Morelos, Cuernavaca, Instituto de Cultura de Morelos/México, Conaculta/M. A. Porrúa, 1997.

NicColai, Sergio y Humberto Morales Moreno (coords.), La cultura industrial mexicana. Primer encuentro nacional de arqueología industrial. Memoria (Monterrey, N. L., 3-5 de junio de 1999), Puebla, Benemérita Universidad Autónoma de Puebla/ Comité Mexicano para la Conservación del Patrimonio Industrial, 2003.

NiCKel, Herbert J., Henequén-Plantagen in Yucatán. Vom Ende einer agro-industriellen Monokultur in Mexiko. Eine Dokumentation in Bildern, Friburgo, Arnold Bergstraesser Institut, 1995.

Novelo, Victoria (coord.), Arqueología de la industria en México, México, Museo Nacional de Culturas Populares, s. f. [1984].

Ortiz Hernán, Sergio (coord.), De las estaciones, Puebla, Museo Nacional de los Ferrocarriles Mexicanos/México, Secretaría de Comunicaciones y Transportes/Ferrocarriles Nacionales de México, 1995.

Samaran, Chardes (dir.), L'histoire et ses méthodes, París, Gallimard, 1961.

Sande, Theodore Anton, Industrial Archaeology. A New Look at the American Heritage, Nueva York, Penguin Books, 1978.

Stratton, Michael y Barrie Trinder, Book of Industrial England, Londres, B. T. Batsford/English Heritage, 1997.

, Twentieth Century Industrial Archaeology, Londres/Nueva York, E \& FN Spon, 2000.

TAVONe, Francesco, "Introduzione" en BernardetTa Ricatti y FranCesco Tavone (coords.), Archeologia industriale e scuola, Florencia, Manzuoli/Casale Monferrato, Marietti, 1989, pp. 1-3.

Tognarini, IVAN y ANGelo Nesti, Archeologia industriale. L'oggetto, i metodi, le figure professionali, Roma, Carocci, 2003.

TOURING Club ITALIANo y ASSOCIAZIONe Museimpresa, Turismo industriale in Italia. Arte, scienza, industria, un patrimonio culturale conservato in musei e archivi d'impresa, Milán, Touring Editore, 2003. 
Trinder, BARrie (ed.), The Blackwell Encyclopedia of Industrial Archaeology, Oxford/Cambridge, Mass., Basil Blackwell, 1992, 2 tomos.

VEGETTI, MARIO, "Le scienze della natura e dell'uomo nel v secolo" en Ludovico Geymonat, Storia del pensiero filosofico e scientifico, Milán, Garzanti, 2a. ed., 1988, vol. 1, pp. 110-150. 


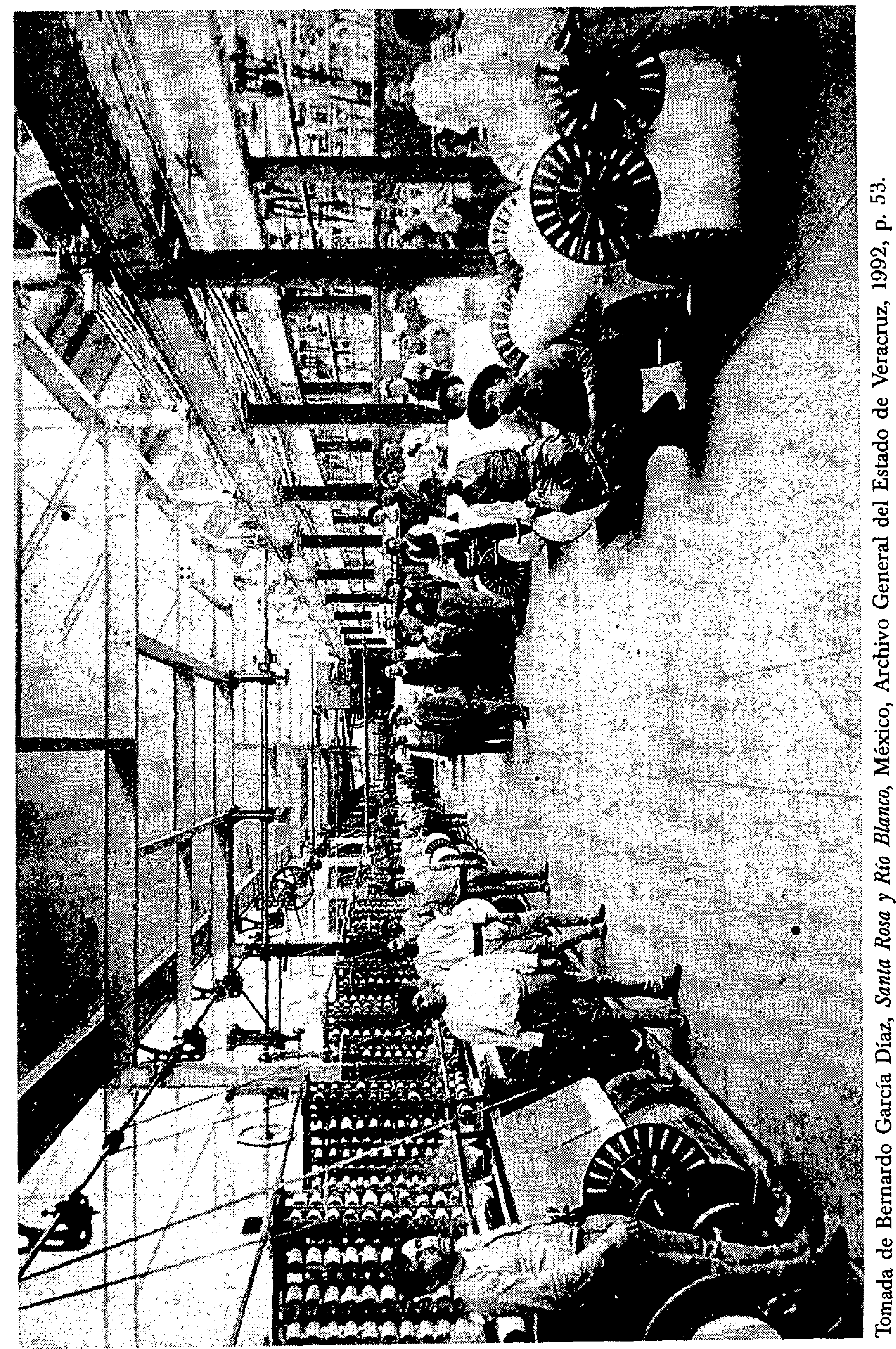

